\title{
Electronic Structure of Small Surfactants: a Continuum Solvation Study
}

\author{
Krzysztof Mozgawa and Luca Frediani* \\ Centre for Theoretical and Computational Chemistry, Department of Chemistry, UiT, The \\ Arctic University of Norway, N-9037 Tromsø, Norway \\ E-mail: luca.frediani@uit.no \\ Phone: +47 77640785
}

\begin{abstract}
The effect of surfaces and interfaces on the structure and properties of molecules is of a great interest in a number of biological and technological applications. Nevertheless, such an investigation is extremely challenging from an experimental point of view, due to the bidimensionality of the environment. In recent years, we have developed a framework to study molecules at surfaces and interfaces, by means of a continuum approach. In the present study, we extend our model by showing the effect of interfacial solvation on molecular properties and by refining the description of the transfer process, making use of a proper Boltzmann averaging of the orientational degrees of freedom. Our findings are in substantial agreement with previous simulations, for the energetics of the transfer process, but yield a somehow different description of the molecular electronic structure. Both the agreement on the energetics and the disagreement on the properties are motivated in light of the role played by non-electrostatic effects in the solvation process.
\end{abstract}




\section{Introduction}

Dielectric Continuum (DC) methods are nowadays routinely implemented in most quantum chemistry software packages. They are by far the simplest way to include solvent effects in a quantum chemical calculation: solvent molecules are replaced altogether by a structureless medium, surrounding the target molecule which is enclosed in a cavity. DC models can often be used as a black-box, specifying only a handful of parameters, to define the cavity and the physical properties of the medium. ${ }^{1,2}$

Despite such a simplicity, DC models have advanced significantly from the original spherical-cavity models of Onsager and Kirkwood, ${ }^{3,4}$ and feature molecular-shaped cavities ${ }^{5}$ and the possibility to describe molecular properties to high order, ${ }^{2,6-10}$ they can employ several non-equilibrium schemes to model the delay in the solvent response, ${ }^{8,11-13}$ and have been extended to non-electrostatic interactions. ${ }^{14,15}$ The calculation of solvation energies within a DC framework has become routine, and it yields reliable, quantitative information about the solvation energetics. ${ }^{16,17}$

Such a success has motivated the extension of the original models in several directions, to deal with an ever growing spectrum of physical phenomena. One such development regards the possibility to describe heterogeneous environments where the properties of the solvent are no longer constant in space: this is the natural way for a continuum method, which defines the solvent by its macroscopic properties (permittivity, refractive index and density just to mention the most important ones), to deal with surfaces and interfaces. The importance of such environments is paramount. In technological applications, the miniaturization paradigm clearly exemplified by nanotechnology, implies that a large part of the underlying molecular processes happens in the presence of a surface, such as for example the energy transfer from a dye to the semiconductor in a dye-sensitized solar cell. ${ }^{18}$ On the opposite side of the spectrum are natural environments: the lipidic membranes of a cell ${ }^{19}$ for its biologic implications or 
the surface of the sea ${ }^{20}$ for its environmental impact. The theoretical study of molecular processes at surfaces and interfaces is therefore a very active field of research. However, continuum methods have only sporadically been used in this context. Most often interfacial phenomena are modeled either by fully atomistic simulations, ${ }^{21-23}$ if one is interested in the behavior of single atoms and molecules, or by coarse-grained methods, to describe phenomena at larger time and size scales. ${ }^{24-26}$ Common to both approaches is however the treatment of the whole system at a uniform level (with or without atomistic detail), but even for the atomistic simulations the investigation of molecular properties is precluded due to the lack of a quantum description of the system. On the other hand, molecular properties (dipoles, (hyper)polarizabilities, excitation energies) are the essential link between modeling and the various spectroscopies which are employed to investigate surfaces and interfaces. ${ }^{27}$ Focused models are a good choice to fill this gap: they provide a quantum description of the substrate in presence of the environmental effect, which is included either with atomistic detail, such as in Quantum Mechanics/Molecular Mechanics (QM/MM) calculations, ${ }^{28-30}$ or with an averaged reaction field, such as for continuum models. ${ }^{1,31}$ In 2000, we have presented our first continuum model for surfaces and interfaces using Polarizable Continuum Model (PCM) including all relevant solvation energy contributions, ${ }^{32}$ albeit most of them were only included empirically (no quantum description). Later we have presented an improved version of the quantum electrostatic model, ${ }^{33}$ and we have gradually included the other components such as repulsion ${ }^{34}$ and dispersion ${ }^{35}$ in the quantum model. At present, all relevant terms besides cavitation are defined at the Quantum Mechanics (QM) level, thus their effect on the electronic density and in turn on molecular properties is included. Lately also a QM/MM based approach has been employed by Martins-Costa and coworkers, ${ }^{36-39}$ where MD simulations are combined with a $\mathrm{QM} / \mathrm{MM}$ treatment of the core to retain a quantum picture of the solute.

The main challenge for continuum models, in this context, is the lack of specific solute- 
solvent interactions which are instead present in QM/MM calculations. The advantage is however a significantly lower computational cost.

The main goal of this manuscript is to assess the validity of our current model, by applying it to some test-system, and compare the obtained results to the corresponding QM/MM simulations performed by Martins-Costa and coworkers. We will first outline our model, with emphasis on the aspects which are related to the extension to interfaces. Then, we will describe the computational protocol employed, followed by the presentation and discussion of our results. Our main findings will then be summarized in the concluding remarks..

\section{Theory}

In this section we will present the theoretical foundation of our model. We start from the definition of the solvation process as the transfer of an isolated molecule from the gas-phase to the solution, and the required adaptations to describe interfacial environments.

At constant pressure and temperature, the correct thermodynamic quantity is the Gibbs' free energy $G^{\text {sol }}$. which is the net work required to create a cavity within the solvent and to bring the two non-interacting systems (solute and solvent) to the fully interacting state. Following Tomasi et al. ${ }^{2}$ we divide such a work in four additive contributions: $G^{e l}, G^{\text {dis }}, G^{\text {rep }}$ and $G^{c a v}$, which are the electrostatic, dispersion, repulsion and cavitation contributions to free energy of solvation, respectively. We neglect in this work the vibrational contribution to the free energy.

In our model $G^{e l}, G^{d i s}, G^{r e p}$ are included in the quantum Hamiltonian, whereas $G^{c a v}$ is only treated classically. While not included in our model, quantum approach to cavitation has been recently presented by Andreussi et al. ${ }^{40}$

In the following we will first consider our approach to deal with surfaces and interfaces. We will then review each solvation energy contributions briefly, emphasizing aspects which 
are relevant in connection to the extension to surfaces and interfaces.

\section{Interfaces}

In continuum solvation methods, the solvent structure is largely discarded: the solvent parametrization is reduced to a handful of parameters which are necessary to model the relevant solute-solvent interactions. For uniform and homogeneous solvents these parameters are scalars, such as the dielectric constant (electrostatics), the refractive index (dispersion), the density of valence electrons (repulsion), the surface tension (cavitation). In some cases, such as liquid crystals, a tensorial permittivity is used to model the non-uniformity of the electrostatic response along different spacial directions. ${ }^{41}$ In the presence of surfaces and interfaces, the homogeneity is no longer valid, and it is necessary to introduce positiondependent parameters. The simplest choice is the subdivision of the environment in different, boundary-separated domains. Despite its formal simplicity, such a solution is technically challenging: the parametrization of interfaces alongside the molecular cavity is difficult to achieve, especially for infinite boundaries such as planar ones, and it leads to unphysical artifacts like the singularity due to image charges, when the molecular cavity is brought very close to the interface. A better alternative is achieved by allowing solvent descriptors to be smooth, position dependent-functions: the topology of the environment is hidden in such functions and unphysical singularities are avoided.

In our model, for each physical parameter $\xi$ employed in the working equations, we have made use of a simple parametrization, where for each point in space $\xi$ is a weighed average of the bulk values for the solvents on the two sides of the interface. For instance, for a planar interface perpendicular to the $z$ axis, a generic solvent property $\xi(z)$ is parametrized as:

$$
\xi_{M}=\xi_{1} x(z)+\xi_{2}(1-x(z)) .
$$


For a solvent-solvent interface the weights can be considered the molar fractions of the two solvents, computed by molecular dynamic simulations. ${ }^{42-44}$ For solvent/vapor surfaces it is instead more appropriate to consider a dimensionless density fraction: $x(z)=\rho(z) / \rho_{0}$, where $\rho_{0}$ is the density in the bulk.

In practice, for planar surfaces (both solvent/solvent and solvent/vapor ones), $x(z)$ is chosen to have the following form:

$$
x=\frac{1}{2}+\frac{1}{2} \tanh \frac{z-z_{0}}{W / 6},
$$

where $z_{0}$ is the midpoint of the interface, and $W$ is a width parameter. The constant factor 6 determines a dilation of the profile such that $W$ corresponds roughly to a $3 \sigma$ interval, if the shape of the profile is interpreted as an integrated statistical distribution, and $\sigma$ is its standard deviation.

A question arising from the choice of profile is how to deal with quantities which are related to each other. For instance, the refractive index $\eta$ is connected to the optical permittivity: $\epsilon_{\infty}=\eta^{2}$. In principle, one of them could be derived from the other throughout the interface. On the other hand, such a level of sophistication is unjustified and a more pragmatic approach has been used. For each contribution to the solvation energy, we have identified a phenomenological parameter which is varied through the interface according to Eq. (1). In Table 1, we have summarized the parameters employed, their definition and we have listed which energy contribution they are used for.

\section{Electrostatics}

The electrostatic term is certainly the most important among the solute-solvent interactions: in the vast majority of implementations it is actually the only one considered. We compute the electrostatic component $\Delta G_{e l}$ by making use of the Integral Equation Formal- 
Table 1: Phenomenological parameters used in the different contributions to the solvation free energy and their values for the solvents used in this study. For electrostatics, the dielectric constant $\epsilon$ is employed; for dispersion the coefficient $\beta=c_{f} \frac{\eta_{S}^{2}-1}{\eta_{s}^{2}} \cdot \frac{\Omega^{S}}{\Omega^{S}+\omega_{a v e}}$ is employed, containing both solvent-dependent parameters (a fitting constant $c_{f}$, the refractive index $\eta$ and the ionization energy $\Omega$ ) and an average excitation energy for the solute $\omega_{\text {ave }}$; for repulsion, the relevant quantity is the energy density of valence shell electrons of the solvent $\rho$ (in Hartree); for cavitation, the surface tension $\gamma\left(\right.$ in $\left.10^{-3} N \cdot m\right)$ is employed.

\begin{tabular}{l|c|rrr} 
Component. & Quantity & Water & $\mathrm{CCl}_{4}$ & Vacuum \\
\hline \hline Electrostatics & $\epsilon$ & 78.39 & 2.228 & 1.0 \\
Dispersion & $\beta$ & (system dep.) & (system dep.) & 0.0 \\
Repulsion & $\rho$ & $9.37 \cdot 10^{4}$ & $5.11 \cdot 10^{5}$ & 0.0 \\
Cavitation & $\gamma$ & 71.81 & 26.15 & 0.0
\end{tabular}

ism (IEF): ${ }^{41,45}$ the problem is recast into a boundary-integral problem at the cavity surface,

$$
A \sigma=-g
$$

where $A$ is an integral operator, $\sigma$ is the apparent surface charge on the cavity surface and $g$ is function supported on the cavity surface.

More in detail we have that:

$$
\begin{aligned}
& A=\left(2 \pi-D_{e}\right) S_{i}+S_{e}\left(2 \pi+D_{i}^{*}\right) \\
& g=\left(2 \pi-D_{e}\right) V_{M}+S_{e}\left(\frac{\partial V_{M}}{\partial n}\right)
\end{aligned}
$$

where $V_{M}$ is the electrostatic potential generated by the solute charge distribution, and $D_{a}$ and $S_{a}$ ( $a=i, e$, for inside and outside the cavity, respectively) are also integral operators defined as

$$
\begin{gathered}
\left(S_{a} \sigma\right)(x)=\int_{\Gamma} G_{a}(x, y) \sigma(y) \mathrm{d} y \\
\left(D_{a} \sigma\right)(x)=\int_{\Gamma}\left[\epsilon_{a}\left(\vec{\nabla}_{y} G_{a}(x, y)\right) \cdot \vec{n}(y)\right] \sigma(y) \mathrm{d} y
\end{gathered}
$$




$$
\left(D_{a}^{*} \sigma\right)(x)=\int_{\Gamma}\left[\epsilon_{a}\left(\vec{\nabla}_{y} G_{a}(x, y)\right) \cdot \vec{n}(x)\right] \sigma(y) \mathrm{d} y
$$

and $G_{a}$ denotes a Green's function. $\Gamma$ is the cavity surface, $\epsilon_{a}$ is the medium permittivity (equal to 1 for $a=i$ ), and $\vec{n}$ is the outward-pointing unit vector perpendicular the the surface of the cavity. The main strength of the IEF-PCM is the possibility to deal with any medium, as long as the Green's function is either known in a closed form or available through numerical integration. Standard solvents, nematic phases, ionic solutions ${ }^{45}$ and sharp planar interfaces $^{32}$ belong to the first group, whereas diffuse interfaces ${ }^{33}$ and spherical systems ${ }^{46}$ belong to the second.

As an example of the first class, we consider a uniform, homogeneous dielectric whose Green's function can be written as

$$
G_{i}(x, y)=\frac{1}{\epsilon|x-y|}
$$

where $\epsilon$ is the permittivity of the medium. For the cavity interior vacuum is considered $(\epsilon=1)$.

On the other hand, for the diffuse interfaces, ${ }^{33}$ a closed-form expression cannot in general be found, but it can be computed numerically as the solution of the following differential equation where $\epsilon(x)$ becomes position-dependent.

$$
\vec{\nabla}_{x} \cdot\left[\epsilon(x) \cdot \vec{\nabla}_{x} G^{E}(x, y)\right]=-4 \pi \delta(x-y)
$$

In case of a planar diffuse interface the permittivity is dependent on $z$, allowing for a simple numerical integration. ${ }^{33}$ The final expression consists of two terms, representing the direct Coulomb interaction, mediated by an effective permittivity $c(x, y)$ and by an image 
term $G_{\text {img }}$ which corresponds to the image-charge potential for a sharp planar interface:

$$
G_{e}(x, y)=\frac{1}{c(x, y)|x-y|}+G_{\mathrm{img}}(x, y)
$$

\section{Dispersion}

In the theory of intermolecular interactions, ${ }^{47,48}$ dispersion arises from the interaction between transition charge densities of the two interacting subsystems. For a solute-solvent system, one such subsystem is represented by the solute, whereas the other one is the solvent. A strategy to extend dispersion to such systems has originally been devised by Amovilli, ${ }^{49}$ and then implemented by Amovilli and Mennucci ${ }^{15}$ and later modified by Weijo et al. ${ }^{50} \mathrm{In}$ such a model, the free energy of solvation is computed through the following expression

$$
G^{d i s p}=-\frac{1}{8 \pi} \frac{\eta_{S}^{2}-1}{\eta_{S}^{2}} \sum_{p} \frac{\Omega^{S}}{\Omega^{S}+\omega_{p}} \int_{\Gamma} \mathrm{d} s V_{p}(s) E_{p}(s)
$$

where the sum runs over the excited states of the solute. $V_{p}$ and $E_{p}$ are, respectively, the electrostatic potential and electrostatic field connected to the transition charge density of state $p, \omega_{p}$ is the corresponding excitation energy and $\Omega^{S}=I_{s} \eta_{s}$ is the product of solvent first ionization potential $I_{s}$ and its refractive index $\eta_{S}$. Although Eq. 12 can be employed to compute the dispersion energy, it is impractical to obtain the corresponding Fock operator. Therefore, a simplified expression is employed, assuming an average excitation energy $\omega_{\text {ave }}$ instead of $\omega_{p}$. Additionally, the factor $\frac{1}{8 \pi}$ is replaced by a solvent-dependent coefficient $c_{f}$ which is fitted for each solvent:

$$
G^{d i s p}=-c_{f} \frac{\eta_{S}^{2}-1}{\eta_{S}^{2}} \frac{\Omega^{S}}{\Omega^{S}+\omega_{\mathrm{ave}}} \int_{\Gamma} \mathrm{d} s \sum_{p} V_{p}(s) E_{p}(s)=-\beta\left(\omega_{\mathrm{ave}}\right) \int_{\Gamma} \mathrm{d} s \sum_{p} V_{p}(s) E_{p}(s) .
$$

The value of $\omega_{\text {ave }}$ is obtained by imposing that Eq. 12 and Eq. 13 yield the same disper- 
sion energy. The extension to the interfaces ${ }^{35}$ is simply obtained by collecting all solventdependent parameters in a coefficient $\beta$ :

$$
\beta=c_{f} \frac{\eta_{S}^{2}-1}{\eta_{S}^{2}} \frac{\Omega^{S}}{\Omega^{S}+\omega_{\mathrm{ave}}}
$$

which is then made position-dependent. The final expression for free energy of dispersion at the diffuse interface is then as follows:

$$
G^{d i s p}=-\int_{\Gamma} \beta\left(\omega_{\text {ave }} ; s\right) V_{p}(s) E_{p}(s) \mathrm{d} s
$$

where the position dependence of $\beta$ is given by Eq. (1).

\section{Repulsion}

The solute electronic density is not entirely confined within the cavity boundary. The outlying charge has a repulsive interaction with the solvent charge density which arises from the Pauli principle. ${ }^{47,48}$ The repulsion contribution to the solvation free energy ${ }^{15}$ can be written as

$$
G_{r e p}=\alpha \int_{r \notin C} d r P_{A}(r),
$$

where the integral is over the whole space except the cavity, $P_{A}(r)$ is the electronic density (where $A$ refers to the solute), and $\alpha$ is a constant factor, proportional to the density $\rho_{e}$ of valence shell electrons in the solvent:

$$
\alpha=0.063 \rho_{e}=0.063 \rho_{B} \frac{n_{v a l}^{B}}{M_{B}},
$$

which is in turn obtained from the solvent density $\rho_{B}$, the number of valence shell electrons $n_{v a l}^{B}$, and the molecular weight of the solvent $M_{B}$. 
For diffuse interfaces, it suffices to make the density of valence-shell electron position dependent:

$$
G_{r e p}=\alpha^{\prime} \int_{r \notin C} \rho_{e}(r) P_{A}(r) \mathrm{d} r,
$$

where $\alpha^{\prime}=0.063$. In order to avoid a costly volume integral, we assume an exponential decay of the electronic density outside the cavity. For a spherical cavity, the problem admits a simple analytical integration (see Ref. ${ }^{34}$ for details), leading to a surface integral which requires a weight function $f(s ; b)$ depending on an adjustable parameter $b^{\text {a }}$, the solute electronic density at the cavity surface $P_{A}(s)$ and the density of valence shell electron of the solvent at the surface $\rho_{e}(s)$ :

$$
G_{r e p}=\alpha^{\prime} \int_{s \in \Gamma} \rho_{e}(s) f(s ; b) P_{A}(s) \mathrm{d} s,
$$

The expression above is then used with an arbitrary cavity, simply by adjusting $b$ such that the outlying electronic charge $n_{\text {out }}$ is correctly reproduced:

$$
n_{\text {out }}=\int_{s \in \Gamma} f(s ; b) P_{A}(s) \mathrm{d} s .
$$

\section{Cavitation}

Cavitation is the energy required to create a cavity inside the solvent. Therefore, it is only indirectly dependent on the solute, through the cavity shape. Scaled-Particel Theory (SPT), - a statistical thermodynamics formalism developed by Pierotti ${ }^{51}$ for spherical cavities and a hard-sphere liquids - is employed here. This method has been adapted to molecular-shaped cavities by adding the cavitation contributions from each sphere, and weighing each of them

\footnotetext{
${ }^{a}$ In Ref. ${ }^{34}$ the symbol $\beta$ is employed. Here, $b$ is employed, to avoid confusion with the dispersion parametrization.
} 
by their respective solvent-exposed fraction.

$$
G_{c a v}=\sum_{n} \omega_{n} G_{c a v}\left(R_{n}\right)
$$

where $R_{n}$ is the radius of the sphere $n$, and $\omega_{n}$ is the weight. To extend model to surfaces and interfaces, it is convenient to rewrite the expression in terms of each tessera

$$
G_{c a v}=\sum_{t} \frac{a_{t}}{4 \pi R_{t}^{2}} G_{c a v}\left(R_{t}\right)
$$

where $a_{t}$ is the area of the tesserae, and $R_{t}$ is the radius of the sphere; the cavitation free energy is then split in two terms, one from each solvent:

$$
G_{c a v}=\sum_{t} x_{t} \frac{a_{t}}{4 \pi R_{t}^{2}} G_{c a v}^{1} R_{t}+\left(1-x_{t}\right) \frac{a_{t}}{4 \pi R_{t}^{2}} G_{c a v}^{2} R_{t}
$$

where $x_{t}$ and $\left(1-x_{t}\right)$ weigh the contributions of each solvent. For sharp planar interfaces $x_{t}=1$ if the tessera $t$ is inside the first solvent and zero otherwise. The drawback is that the cavity generation must also split tesserae at the interface in two parts, each belonging to a different solvent. For diffuse interfaces, it is instead only required that $x_{t}=x\left(s_{t}\right)$, where $x(s)$ is a smooth function of the position, defining the transition from one solvent to the other.

Additionally, to model surfactant behavior, we have introduced a surface term. When the cavity is moving through the interface, it reduces the solvent surface thus lowering the energy by ${ }^{35}$

$$
G_{\text {surf }}=-\gamma_{s} A(z=0)
$$

where $\gamma_{s}$ is the surface tension, and $A(z=0)$ is the cross section of the cavity in the plane 
of the interface. For a diffuse interface $A$ is calculated as ${ }^{35}$

$$
A=\int_{\Gamma}\left(\rho(z) / \rho_{0}-\frac{1}{2}\right) \hat{z}(s) \cdot \hat{n}(s) d s .
$$

\section{Contributions to the Fock operator}

All contributions to the Fock operator can be obtained as the first derivative with respect to the density matrix elements of the corresponding free energy contribution. The extension to surfaces and interfaces does not pose any problem, because the interface parametrization is independent of the density matrix. All such expressions have been derived elsewhere and we will here limit ourselves to summarizing them in a uniform notation.

Electrostatics contains both a monoelectronic and a bielectronic term. The corresponding Fock operator elements can therefore be written as:

$$
F_{\mu \nu}^{e l}=\frac{\partial G}{\partial P_{\mu \nu}}=\left(\sigma_{N}+\sigma_{e}\right) \cdot V_{\mu \nu}
$$

where the monoelectronic term contains the Apparent Surface Charge (ASC) generated by the nuclei $\left(\sigma_{N}\right)$ and the bielectronic one contains the ASC from the electrons.

Repulsion is instead a monoelectronic term. We report here the expression derived in Ref. ${ }^{34}$ which can be used in the presence of interfaces

$$
F_{\mu \nu}^{r e p}=\frac{\partial G^{r e p}}{\partial P_{\mu \nu}}=\alpha^{\prime} \int_{\Gamma} \rho_{e l}(s) f(s) \delta_{\mu \nu}(s) \mathrm{d} s
$$

where $\delta_{\mu \nu}(s)$ is the representation of the delta-function operator such that $\operatorname{Tr} \boldsymbol{P} \boldsymbol{\delta}(s)=\rho(s)$. Dispersion also contains a monoelectronic and a bielectronic contribution:

$$
F_{\mu \nu}^{d i s}=-\sum_{\xi \eta}[\mu \xi \mid \nu \eta]\left(S_{\xi \eta}^{-1}-P_{\xi \eta}\right)
$$


where $S$ and $P$ are the overlap and density matrices, and

$$
[\mu \xi \mid \nu \eta]=\int_{\Gamma} \beta(s)\left[V_{\mu \xi}(s) E_{\nu \eta}+V_{\nu \eta} E_{\mu \xi}(s)\right] \mathrm{d} s .
$$

Cavitation is the only term which do not contribute to the Fock matrix, because it is independent of the electronic degrees of freedom.

\section{Contributions to the linear response equations}

Response properties, in a Time-Dependent Density Functional Theory (TDDFT) framework are computed by solving a set of coupled linear equations:

$$
\boldsymbol{G}^{[2]}=\left(\begin{array}{cc}
\boldsymbol{A} & \boldsymbol{B} \\
\boldsymbol{B}^{*} & \boldsymbol{A}^{*}
\end{array}\right) \cdot\left(\begin{array}{c}
\boldsymbol{X} \\
\boldsymbol{Y}
\end{array}\right)=\omega\left(\begin{array}{cc}
\boldsymbol{1} & 0 \\
0 & -\mathbf{1}
\end{array}\right)=\left(\begin{array}{c}
\boldsymbol{X} \\
\boldsymbol{Y}
\end{array}\right)
$$

where the matrices $\boldsymbol{A}$ and $\boldsymbol{B}$ collect the second derivatives of the free energy functional with respect to orbital rotations. It is beyond the scope of the present paper to expose the complete derivation and the reader is refereed to the relevant literature on the subject. ${ }^{52,53}$ We limit ourselves to consider the terms which arise due to presence of the solvent.

Of the four contributions mentioned above, three of them depend on the electronic density: electrostatics, dispersion and repulsion. The former two contain mono- and bielectronic terms (the bielectronic terms are products of monoelectronic ones). Cavitation is on the other hand only dependent on the cavity shape and not directly on the density.

Any monoelectronic contribution to the solvation energy can be formally written as:

$$
G_{1}=\int F_{1}(r) \rho(r) \mathrm{d} r
$$

where $F_{1}(r)$ is the corresponding energy functional. Conversely, any bielectronic contribution 
to the solvation energy can be written as:

$$
G_{2}=\int \rho\left(r^{\prime}\right) F_{2}\left(r, r^{\prime}\right) \rho(r) \mathrm{d} r
$$

where $F_{2}(r)$ is the corresponding bielectronic energy functional. In particular the matrix $\boldsymbol{A}$ and $\boldsymbol{B}$ are obtained as:

$$
\begin{aligned}
& \boldsymbol{A}_{a i, b j}=\frac{\partial^{2} G}{\partial \kappa_{a i}^{*} \partial \kappa_{b j}}=\frac{\partial G}{\partial \rho} \frac{\partial^{2} \rho}{\partial \kappa_{a i}^{*} \partial \kappa_{b j}}+\frac{\partial^{2} G}{\partial \rho^{2}} \frac{\partial \rho}{\partial \kappa_{a i}^{*}} \frac{\partial \rho}{\partial \kappa_{b j}} \\
& \boldsymbol{B}_{a i, b j}=\frac{\partial^{2} G}{\partial \kappa_{a i}^{*} \partial \kappa_{b j}^{*}}=\frac{\partial G}{\partial \rho} \frac{\partial^{2} \rho}{\partial \kappa_{a i}^{*} \partial \kappa_{b j}^{*}}+\frac{\partial^{2} G}{\partial \rho^{2}} \frac{\partial \rho}{\partial \kappa_{a i}^{*}} \frac{\partial \rho}{\partial \kappa_{b j}^{*}}
\end{aligned}
$$

In practice the first term of the expressions for $\boldsymbol{A}$ and $\boldsymbol{B}$ is already present in the Fock operator and only the second term needs to be considered explicitly. Additionally, only the two-electron terms contribute to $\partial^{2} G / \partial \rho^{2}$. For the electrostatic part such a contribution can be written as:

$$
A_{2, a i, b j}^{e l}=\int_{\Gamma} \sigma_{a i}(s) V_{b j}(s) \mathrm{d} s
$$

whereas for dispersion one gets

$$
A_{2, a i, b j}^{d i s}=\int_{\Gamma} \beta(s) E_{a i}(s) V_{b j}(s) \mathrm{d} s .
$$

The contributions to $\boldsymbol{B}$ are very similar and their derivation is not reported here.

Following Cupellini et al. ${ }^{54}$ we have adjusted the contribution of dispersion to the response equation by multiplying $\beta(s)$ (see Eq. 14)) by a factor 1.51, to obtain better agreement for the calculation of excitation energies. 


\section{Computational protocol}

For all calculations, including all geometry optimizations, we have employed the B3LYP functional ${ }^{55,56}$ as implemented in the Gaussian $09^{57}$ program. For all the systems, the augcc-pVDZ ${ }^{58}$ basis set has been employed.

All geometries were optimized in water. No reoptimization during solvation profile generation was done. In all calculations the free energy has been obtained without inclusion of thermal corrections (vibrational and rotational terms).

We have constructed molecular cavities using interlocking, atom-centered spheres and the following set of radii: $1.6 \AA$ for nitrogen, $1.2 \AA$ for hydrogens capable of hydrogen bonding $\left(\mathrm{OH}, \mathrm{NH}_{2}\right) 1.52 \AA$ for oxygen, and $1.8 \AA$ for carbon. For $\mathrm{CH}, \mathrm{CH}_{2}$ and $\mathrm{CH}_{3}$ groups, a common sphere centered on carbon has been employed with radii of $1.9 \AA, 2.0 \AA$ and $2.1 \AA$ respectively. All radii are multiplied by the common scaling factor of 1.2. No additional spheres to account for further solvent excluded volume have been used.

In the presence of an interface, the solvation free energy becomes dependent on the position and orientation of the molecule with respect to the interface. In MD simulations such an effect is reflected in the potential of mean force where orientations at lower energy will naturally contribute more, according to the corresponding Boltzmann distribution. In our case, where each calculation is performed at fixed position and orientation, we have performed a Boltzmann averaging following the procedure described by Parisio and Ferrarini. ${ }^{59}$ In particular, we are interested in the orientationally averaged value of a given quantity $f$, which can be expressed as:

$$
\langle f\rangle_{\Omega}(z)=\frac{\int f(z, \Omega) e^{-U(z, \Omega) / k_{b} T} \mathrm{~d} \omega}{e^{-U(z, \Omega) / k_{b} T} \mathrm{~d} \omega}
$$

where $f(z, \Omega)$ is the value of the quantity at a fixed distance $z$ of the center of mass of the molecule from the interface and a given molecular orientation $\Omega$ with respect to the interface, 
and $U(z, \Omega)$ is the corresponding solvation energy. If a Leopardi's sampling ${ }^{60}$ is employed the orientational average can be expressed as:

$$
\langle f\rangle_{\Omega}(z)=\frac{\sum_{i} f\left(z, \Omega_{i}\right) e^{-U\left(z, \Omega_{i}\right) / k_{b} T}}{\sum_{i} e^{-U\left(z, \Omega_{i}\right) / k_{b} T}}
$$

because each representative point has the same weight due to the chosen partitioning scheme.

\section{Results discussion}

In our study, we have considered the following substrates: formaldehyde, benzaldehyde and o-cyanophenol at the water-vapor interface, valine and glycine (in neutral and zwitterionic forms) at the water $/ \mathrm{CCl}_{4}$ interface. They are among the systems which have recently been investigated by Martins-Costa and coworkers, ${ }^{36-38,61}$ and have been chosen to compare results across different theoretical models. See Ref. ${ }^{36,37}$ for aldehydes, Ref. ${ }^{61}$ for $o$-cyanophenol, and 38 for the aminoacids. For each compound we will illustrate our results, drawing a comparison with the above mentioned studies.

\section{Formaldehyde}

In Fig. 1 we have reported several properties of formaldehyde crossing the water surface, obtained with our model. The free energy of solvation, is shown in Fig. 1a. The bulk solvation energy in water is $-1.66 \mathrm{kcal} / \mathrm{mol}$. Approaching the interface, the solvation energy becomes larger (more negative), passing through a minimum at 0 Angstrom, which is about $2 \mathrm{kcal} / \mathrm{mol}$ lower than the bulk value. Thereafter, the solvation energy increases towards zero (gas phase).

As observed before ${ }^{32,35}$ the insurgence of the minimum is due to competing effects between negative contributions to the solvation energy (electrostatics, dispersion, surface term) and 
positive ones (repulsion and cavitation), which arise at different positions and with different slopes across the interface (Fig. 1b). In particular, going from vacuum to water, electrostatics is already present at -5 Angstrom, which is consistent with its long-range nature, whereas the others arise slightly later. In summary, the electrostatic part of the free energy is the main driving force for the solvation process, whereas the non-electrostatic contributions are responsible for the interfacial behavior, generating the corresponding free energy minimum.

By comparing our results with the findings of Martins-Costa et al, ${ }^{37}$ (see the insert in Fig 1a), we see that the two profiles are very similar, both in terms of shape and magnitude of the free energy profile. In order to confirm the similarity we have also computed the angular distribution of formaldehyde in bulk solution and at the free energy minimum along $z$. Our results are reported in Fig 1c (insert reproduced from Ref. ${ }^{37}$ ). The bulk results show that the normal $\frac{1}{2} \sin (\theta)$ distributions is recovered ${ }^{\mathrm{b}}$, whereas the interfacial distribution peaks at roughly $80^{\circ}$, showing a clear preference for the carbonyl group pointing towards the water surface. The overall shape is also strikingly similar to Ref. ${ }^{37}$

In order to extend the comparison to molecular properties, the dipole, moment, the first five excitation energies and their respective oscillator strengths have been computed throughout the interface. Additionally, the same calculations for a purely electrostatic solvation, by switching off the other energy contributions, have been performed.

In Table 2 we present a summary of our results, comparing our full model (all solvation contributions) to the electrostatic one (dispersion, repulsion and cavitation switched off) and to the results of Ref. ${ }^{37}$ The dipole moment shows similar trends in our calculations with marginal differences when non-electrostatic effects are removed. The comparison with QM/MM results shows also a similar trend, albeit the environmental effect seems enhanced for $\mathrm{QM} / \mathrm{MM}$. It is interesting to note that the HOMO-LUMO gap shows different trends

\footnotetext{
${ }^{b}$ the small discrepancies observed are due to numerical noise in the calculation of solvation free energy with different orientation, because the cavity parametrization is orientation-dependent
} 
with or without non-electrostatic solvation terms: in particular the QM/MM results seems to be in closer agreement with our electrostatic model. This observation applies to excitation energies as well.

Both the similarities and the differences between our model and the simulations in Ref. ${ }^{37}$ can be rationalized in light of the different interactions involved. Our model contains both electrostatic and non-electrostatic contributions: the latter ban be switched on and off to

check their effects on energy and properties. The simulations in Ref. ${ }^{37}$ include electrostatic and non-electrostatic terms in the Potential of Mean Force (PMF), but the QM/MM model employed for molecular properties (dipole moments, HOMO-LUMO gap, excitation energies) is purely electrostatic.

Solvation energies are in good agreement because both models contain electrostatic as well as non-electrostatic interactions. When non-electrostatic interactions are switched off in our model, the minimum at the interface disappears. The dipole moment is practically insensitive to non-electrostatic terms (see Fig. 1d), and our model agrees therefore with QM/MM, which is purely electrostatic. The HOMO-LUMO gap is instead very sensitive to dispersion (see Fig. 1e): only by switching the non-electrostatic terms off we recover the QM/MM results in this case. Similar considerations apply to excitation energies, albeit the sensitivity to dispersion and repulsion is in this case dependent on the specific excitation considered.

\section{Benzaldehyde}

The results for benzaldehyde crossing the water surface are reported in Fig. 2. The free energy profile for benzaldehyde together with the sum of the non-electrostatic contributions (Fig 2a), shows again the importance of the complete picture: the solvation free energy has a minimum around $z=0 \AA$ with a value of $-7.35 \mathrm{kcal} / \mathrm{mol}$. The bulk free energy of solvation is found to be $-3.8 \mathrm{kcal} / \mathrm{mol}$, and the interfacial preference is due to the interplay 
Table 2: Selected molecular properties for formaldehyde at the water surface using full model ('full'), only electrostatic model ('ele'), together with reference ('ref'). ${ }^{37}$ Dipole moments $(\mu$, Debyes), excitation wavelengths $(\lambda \mathrm{nm})$, force constants $\left(f \cdot 10^{3}\right)$, orbital energies (a.u.) of $\operatorname{HOMO}\left(\epsilon_{H}\right)$ and LUMO $\left(\epsilon_{L}\right)$ orbitals.

\begin{tabular}{|lccc|ccc|cc|}
\hline \multicolumn{5}{c|}{ BULK } & \multicolumn{3}{c|}{ INTF. } & GAS \\
\hline & full & ele & ref & full & ele & ref & \multicolumn{3}{c|}{ ref } \\
\hline$\mu$ & 3.030 & 3.043 & $(3.406)$ & 2.966 & 2.973 & $(3.170)$ & 2.446 & $(2.390)$ \\
$\varepsilon_{\mathrm{H}}$ & -0.2755 & -0.2832 & $(-0.2877)$ & -0.2803 & -0.2843 & $(-0.2934)$ & -0.2816 & $(-0.2806)$ \\
$\varepsilon_{\mathrm{L}}$ & -0.0720 & -0.0652 & $(-0.0626)$ & -0.0698 & -0.0666 & $(-0.0699)$ & -0.0670 & $(-0.0642)$ \\
$\varepsilon_{\mathrm{L}}-\varepsilon_{\mathrm{H}}$ & 0.203 & 0.218 & $(0.225)$ & 0.210 & 0.218 & $(0.223)$ & 0.214 & $(0.217)$ \\
$\lambda_{1}$ & 329.9 & 311.5 & $(297.7)$ & 320.7 & 311.9 & $(300.8)$ & 320.7 & $(312.9)$ \\
$f_{1}$ & 0 & 0 & $(0)$ & 0 & 0 & $(0)$ & 0 & $(0)$ \\
$\lambda_{2}$ & 187.0 & 184.6 & $(171.1)$ & 186.1 & 184.8 & $(175.0)$ & 192.0 & $(191.7)$ \\
$f_{2}$ & 10.2 & 10.3 & $(6.8)$ & 12.6 & 12.3 & $(13.4)$ & 27.7 & $(27.8)$ \\
$\lambda_{3}$ & 166.4 & 164.6 & $(154.7)$ & 165.7 & 164.8 & $(157.9)$ & 169.2 & $(170.0)$ \\
$f_{3}$ & 52.6 & 56.3 & $(60.6)$ & 49.3 & 52.0 & $(51.0)$ & 43.9 & $(38.7)$ \\
\hline
\end{tabular}

of the electrostatic and non-electrostatic contributions, arising with different slopes and at different distances from the interface.

The comparison with Ref, ${ }^{36}$ shows a semiquantitative agreement: the position of the minimum is reproduced, and the trends from formaldehyde to benzaldehyde are similar (deeper minimum). The main difference is the width of the interface (intended as the layer where deviations from bulk behavior are observed) which is roughly twice as large when simulations are employed.

When we consider properties, it is also clear that non-electrostatic effects can significantly modify the picture in some cases. The dipole moment is enhanced by the electrostatic interactions, and almost independent of the non-electrostatic terms. In Table 3 we show how removing the non-electrostatic components has no practical effect on the dipole moment both in bulk and at the interface. Compared to Ref. ${ }^{36}$ the agreement is good. The only significant discrepancy is observed at the interface where our values are closer to the bulk than the QM/MM simulations, which is a saturation effect similar to what we have observed 
for formaldehyde.

Turning our attention to the HOMO-LUMO gap, the data from Table 3 show again a significant difference between the full model and the electrostatic one. Following the profile through the interface (see Fig. 2b), the correlation between the trend in the HOMO-LUMO gap and the total solvation energy is clear, showing the importance of non-electrostatic contributions. It is however difficult to draw a comparison with QM/MM calculations, ${ }^{36}$ because the data in bulk are here missing. Excitation energies show a mixed picture, where the solvent shift is sometimes dominated by electrostatics (excitation 1, 3 and 5), and others where dispersion effects are more important (excitation 2 and 4). To illustrate this point, the solvatochromic shifts $\Delta \lambda(z)=\lambda_{\max }(z)-\lambda_{\max , \text { gas }}$ through the interface are reported in Fig. 2c for the first five excitations. To compare our results to Ref., ${ }^{36}$ we have plotted the simulated UV-Vis spectrum in Fig. 2d and reported some selected values in Tab. 4. The main peak $\left(\pi \rightarrow \pi^{\star}\right)$ shows a red-shift at the interface $(13.7 \mathrm{~nm})$ and in bulk $(22.6 \mathrm{~nm})$, in agreement with Ref. ${ }^{36}$ Such an agreement is indeed a consequence of the electrostatic nature of the solvent effect on the $\pi \rightarrow \pi^{\star}$ transition.

It is however not straightforward to compare the $n \rightarrow \pi^{\star}$ transition, in part because of the concurrence of many effects, and in part because in Ref. ${ }^{36}$ only the graphical spectrum with a wider shoulder is reported (it is not possible to conclude from the picture whether a red- or a blue-shift is observed). We therefore limit ourselves to an analysis of the different contributions which are present in our model. For the $n \rightarrow \pi^{\star}$ we observe a small red-shift in bulk $(3.7 \mathrm{~nm})$, whereas we see a blue-shift at the interface $(-6.6 \mathrm{~nm})$. This is reflected in the position of the right shoulder of the spectrum (shifted to the left at the interface and to the right in bulk). ${ }^{\mathrm{c}}$ Without non-electrostatic terms, the $n \rightarrow \pi^{\star}$ transition becomes significantly blue-shifted, whereas non-equilibrium effects are somehow limited. Also for the

\footnotetext{
${ }^{\mathrm{c}} \mathrm{A}$ widening similar to Ref. ${ }^{36}$ cannot be observed because we perform single point calculations and not an averaging over Molecular Dynamics (MD) snapshots.
} 
$\pi \rightarrow \pi^{\star}$ transition we see similar trends: a limited effect due to non-equilibrium solvation and an enhancement of the red-shift when non-electrostatic terms are included. As a last remark we note that our model lacks hydrogen bonding, which is important for the $n \rightarrow \pi^{\star}$ transition. ${ }^{62}$

Table 3: Selected properties of benzaldehyde at the water surface, using the full model (full), the electrostatic model (ele), together with reference values (ref) from Ref. ${ }^{36}$ Dipole moments $\left(\mu\right.$, Debyes), orbital energies (a.u.) of $\operatorname{HOMO}\left(\epsilon_{H}\right)$ and $\operatorname{LUMO}\left(\epsilon_{L}\right)$ orbitals and the HOMO-LUMO gap $\left(\varepsilon_{\mathrm{L}}-\varepsilon_{\mathrm{H}}\right)$, a.u.

\begin{tabular}{|lccc|ccc|cc|}
\hline & \multicolumn{3}{|c|}{ BULK } & \multicolumn{3}{c|}{ INTF. } & \multicolumn{2}{c|}{ GAS } \\
\hline & full & ele & ref & full & ele & ref & \multicolumn{2}{c|}{ ref } \\
\hline$\mu$ & 4.792 & 4.799 & & 4.710 & 4.720 & $(3.96)$ & 3.544 & $(3.56)$ \\
$\varepsilon_{\mathrm{H}}$ & -0.2665 & -0.2724 & -0.2706 & -0.2733 & $(-0.2664)$ & -0.2677 & $(-0.2732)$ \\
$\varepsilon_{\mathrm{L}}$ & -0.0868 & -0.0809 & -0.0850 & -0.0819 & $(-0.0836)$ & -0.0801 & $(-0.0827)$ \\
$\varepsilon_{\mathrm{L}}-\varepsilon_{\mathrm{H}}$ & 0.180 & 0.192 & 0.186 & 0.191 & $(0.183)$ & 0.188 & $(0.191)$ \\
\hline
\end{tabular}

Table 4: $n \rightarrow \pi^{\star}$ and $\pi \rightarrow \pi^{\star}$ excitation energies for benzaldehyde in bulk water, at the water surface and in gas-phase. For each transition, both the absolute value and the solvent shift with respect to gas-phase $(\Delta \lambda)$ are reported. For bulk solvation, both equilibrium and non-equilibrium calculations have been performed, either in combination with the nonelectrostatic terms (full) or without (ele). For the water surface and in gas-phase, we have reported our results together with reference values from Ref. ${ }^{36}$ in parenthesis. All values are in $\mathrm{nm}$.

\begin{tabular}{|c|c|c|c|c|c|c|c|c|c|}
\hline \multicolumn{2}{|c|}{ Excitation } & \multicolumn{4}{|c|}{ BULK } & \multirow{2}{*}{\multicolumn{2}{|c|}{$\frac{\text { INTF. }}{\text { non-eq. }}$}} & \multicolumn{2}{|c|}{ GAS } \\
\hline & & \multicolumn{2}{|c|}{ non-eq. } & \multicolumn{2}{|c|}{ equil. } & & & & \\
\hline & & full & ele & full & ele & full & ref & & ref \\
\hline \multirow{2}{*}{$n \rightarrow \pi^{\star}$} & $\lambda$ & 347.3 & 327.5 & 342.7 & 328.3 & 337.0 & & 343.6 & $(343)$ \\
\hline & $\Delta \lambda$ & 3.7 & -16.1 & -0.9 & -15.3 & -6.6 & & & \\
\hline \multirow[t]{2}{*}{$\pi \rightarrow \pi^{\star}$} & $\lambda$ & 289.7 & 275.6 & 284.9 & 277.8 & 280.6 & $(282)$ & 267.1 & $(274)$ \\
\hline & $\Delta \lambda$ & 22.6 & 8.5 & 17.8 & 10.7 & 13.7 & $(8)$ & & \\
\hline
\end{tabular}

\section{o-cyanophenol}

The results for o-cyanophenol crossing the water surface are reported in Fig. 3. The solvation energy is found to be around $-4 \mathrm{kcal} / \mathrm{mol}$, with a minimum at the interface of about -8 
$\mathrm{kcal} / \mathrm{mol}$. It is again the collective effect of all contributions to the solvation energy, (total and non-electrostatic solvation are reported in Fig. 3a) which determines the surfactant behavior: dispersion $(-18 \mathrm{kcal} / \mathrm{mol}$ in bulk) and cavitation $(17 \mathrm{kcal} / \mathrm{mol})$ are the largest contributions in absolute value, whereas repulsion is positive and small ( $5 \mathrm{kcal} / \mathrm{mol}$ in bulk); electrostatics is slightly larger $(-7 \mathrm{kcal} / \mathrm{mol})$. The surface term, $(-2 \mathrm{kcal} / \mathrm{mol}$ at the surface $)$ contributes to the surfactant behavior, although it is not the only factor (the minimum is present also without the surface term). For this substrate it is more challenging to draw a comparison on the energetics, given that Ref. ${ }^{61}$ only reports an orientational study in the presence of a sharp dielectric surface.

The comparison on simple molecular properties is however illustrative. In Tab 5 we report dipole moment $\mu$, energies of HOMO and LUMO orbitals ( $\varepsilon_{\mathrm{HOMO}}$ and $\left.\varepsilon_{\mathrm{LUMO}}\right)$ for o-cyanophenol, together with the literature values from Ref. ${ }^{61}$ The dipole moments are in good agreement in bulk (4.79 vs, 4.58) and gas-phase (3.67 vs 3.58), whereas our interface values are "saturated" (4.75 vs 3.84). This can be rationalized considering that in Ref. ${ }^{61}$ the interfacial values are only due to the image effect, and the molecule is not partially immersed as in our case. The HOMO-LUMO gap gets instead narrower from gas $(0.189 \mathrm{eV})$ to the interface $(0.182 \mathrm{eV})$ and bulk $(0.176 \mathrm{eV})$ when the full model is employed, whereas no appreciable variation is observed in the electrostatic model $(0.189 / 0.188 / 0.188)$ for the electrostatic model, which agrees very well with Ref. ${ }^{61}$

Table 5: Properties for o-cyanophenol at the water surface using the full model (full), the electrostatic model (ele), together with reference (ref). ${ }^{61}$ Dipole moments $(\mu)$ in Debyes, and orbital energies in a.u.

\begin{tabular}{|lccc|ccc|cc|}
\hline \multicolumn{4}{|c|}{ BULK } & \multicolumn{3}{c|}{ INTF. } & GAS \\
\hline & full & ele & ref & full & ele & ref & \multicolumn{3}{c|}{ ref } \\
\hline$\mu$ & 4.79 & 4.80 & $(4.58)$ & 4.75 & 4.75 & $(3.84)$ & 3.67 & $(3.58)$ \\
$\varepsilon_{\mathrm{H}}$ & -0.2467 & -0.2526 & $(-0.2570)$ & -0.2496 & -0.2526 & $(-0.2649)$ & -0.2576 & $(-0.2610)$ \\
$\varepsilon_{\mathrm{L}}$ & -0.0706 & -0.650 & $(-0.0657)$ & -0.0676 & -0.0649 & $(-0.0733)$ & -0.0683 & $(-0.0679)$ \\
$\varepsilon_{\mathrm{L}}-\varepsilon_{\mathrm{H}}$ & 0.176 & 0.188 & $(0.191)$ & 0.182 & 0.188 & $(0.192)$ & 0.189 & $(0.193)$ \\
\hline
\end{tabular}




\section{Aminoacids}

In Fig. 4, we report free energy of solvation for glycine (left) and valine (right) in the N1 form (top) and in the zwitterionic (middle) as well as a comparison to Ref. ${ }^{38}$ (bottom). All profiles are computed for the $\mathrm{CCl}_{4}$-water interface,

The results for both aminoacids are quite similar and will therefore be discussed in parallel. Both the neutral and the zwitterionic form are preferentially solvated in water (see Fig. 4a-b for the neutral form and Fig. 4c d for the zwitterionic one). The free energy differences are $3 \mathrm{kcal} / \mathrm{mol}$ and $4 \mathrm{kcal} / \mathrm{mol}, 22 \mathrm{kcal} / \mathrm{mol}$ and $27 \mathrm{kcal} / \mathrm{mol}$ glycine-N1, valine-N1, glycine-ZW, and valine-ZW respectively. Moreover, for both molecules the zwitterionic form is always lower than the corresponding neutral form. Both neutral forms display a clear minimum (about $2 \mathrm{kcal} / \mathrm{mol}$ ) at the interface, whereas for the zwitterionic form, where the electrostatic solvation is dominating, such a minimum $(1 \mathrm{kcal} / \mathrm{mol})$ is present only for valine, due to the larger side-chain in this case.

Compared to Ref, ${ }^{38}$ the qualitative agreement is good, because both forms display a similar behavior; however our solvation energy values are smaller in magnitude. To better facilitate the comparison we have translated our profiles to match the PMF plots, by setting to zero the energies in water, as presented in Fig. 4e-f.

In Fig 5 we report the angular distribution for glycine and valine, both in $\mathrm{N} 1$ and ZW forms, at $\mathrm{CCl}_{4}$-water interface and in bulk of $\mathrm{CCl}_{4}$. The reported angle is between the interface normal and the axis bisecting the $C-C-N$ angle where the first carbon belongs to the carboxylic group. At $0^{\circ}$ both polar groups point towards $\mathrm{CCl}_{4}$. As expected, in bulk all profiles roughly reproduce normal distribution of $\frac{1}{2} \sin (\theta)$. The interfacial distribution for the N1 form of glycine shows a more anisotropic distribution with a certain preference for the orientation towards water. In all other cases (ZW for glycine and both forms of valine) there is a clear preference for the polar groups to be oriented towards the water phase, with maxima at $130^{\circ}, 140^{\circ}$ and $160^{\circ}$, for glycine in the $\mathrm{ZW}$ form, and valine in the $\mathrm{N} 1$ form and 
the ZW forms, respectively. The comparison with Ref. ${ }^{38}$ is good for valine in the N1 form, but only qualitatively similar for glycine (N1 form). Angular distributions are not reported in Ref. ${ }^{38}$ for the zwitterionic forms.

\section{Conclusions}

In this study, we have applied the latest development of our continuum model extended to describe solvation at surfaces and interfaces. In order to achieve a correct description of the solvation process, we have included in the model all phenomenologically relevant contributions to solvation: electrostatics, dispersion, repulsion and cavitation. Their extension to surfaces and interfaces has been presented in previous works and has been here summarized in the theoretical section. Our primary concern in this work, was to assess the validity of our model on a set of substrates. To this respect, we have selected systems that have been investigated recently by Martins-Costa and coworkers ${ }^{36-38}$ with a combination of MD simulations to obtain solvation energy profiles as the PMF and QM/MM calculations on selected MD snapshots, to obtain molecular properties at the interface. Additionally we have also

compared our results for o-cyanophenol to the image-charge model presented in Ref., ${ }^{61}$ which is similar to our electrostatic model.

Our results agree well with MD simulations in describing the features of solvation energy profiles. Considering how different the two approaches are, we believe this agreement is a good validation for both models. This conclusion confirms previous results from our group, ${ }^{35}$ and underlines again the importance of non-electrostatic contributions to solvation, when surfaces and interfaces are considered. MD simulations include such contributions by means of a Lennard-Jones potential, whereas QM/MM models lack them in the effective Hamiltonian operator, which is employed for property calculations.

When it comes to molecular properties, we have carefully analyzed the effect of the differ- 
ent contributions (dispersion and repulsion vs. electrostatics) on dipole moments, HOMOLUMO gaps, excitation energies. Our main conclusion is that repulsion and dispersion effects are indeed important, especially for the HOMO-LUMO gap and for selected excitation energies. In this case, to obtain a good agreement with QM/MM simulations, it was necessary to "turn off" non-electrostatic terms, demonstrating that the QM/MM picture is essentially an electrostatic one. On the other hand, an obvious deficiency of our model is the lack of specific interactions, such as hydrogen-bonding which can also have a significant effect at the interface, especially for the $n \rightarrow \pi^{\star}$ transition, such as the one investigated for benzaldehyde.

Both models provide useful insight, because they have complementary strengths and weaknesses: continuum models will need a better description of specific effects, but this is a challenging point for calculations at interfaces insofar as geometry optimizations are not yet available at the interface; QM/MM simulations will require the inclusion of non-electrostatic effects, which are not yet available at the quantum level.

\section{Acknowledgements}

The authors would like to thank Prof. Benedetta Mennucci (University of Pisa) for insightful discussions and suggestions electronic spectra and solvent effects and Dr. Christian S. Pomelli (University of Pisa) for showing us the Leopardi partitioning of the sphere. The computational resources for this project have been provided by the NOTUR high performance computing program (Grant no. NN4654K). This work has been supported by the Research Council of Norway through a Centre of Excellence Grant (Grant No. 179568/V30). The authors also acknowledge financial support by the Troms $\emptyset$ Research Foundation (SURFINT grant). 


\section{References}

(1) Tomasi, J.; Persico, M. Molecular Interactions in Solution: an Overview of Methods Based on Continuous Distributions of the Solvent. Chem. Rev. 1994, 94, 2027-2094.

(2) Tomasi, J.; Mennucci, B.; Cammi, R. Quantum Mechanical Continuum Solvation Models. Chem. Rev. 2005, 105, 2099-3093.

(3) Kirkwood, J. G. Theory of Solutions of Molecules Containing Widely Separated Charges with Special Application to Zwitterions. J. Chem. Phys. 1934, 2, 351-361.

(4) Onsager, L. Electric Moments of Molecules in Liquids. J. Am. Chem. Soc. 1936, 58, $1486-1493$.

(5) Pascual Ahuir, J. L.; Silla, E.; Tunon, I. GEPOL: An Improved Description of Molecular Surfaces. III. A New Algorithm for the Computation of a Solvent-Excluding Surface. J. Comput. Chem. 1994, 15, 1127-1138.

(6) Ferrighi, L.; Frediani, L.; Ruud, K. Excited-State Polarizabilities of Solvated Molecules Using Cubic Response Theory and the Polarizable Continuum Model. J. Chem. Phys. 2010, 132, 024107.

(7) Rizzo, A.; Frediani, L.; Ruud, K. An Ab Initio Investigation of the Buckingham Birefringence of Furan, Thiophene, and Selenophene in Cyclohexane Solution. J. Chem. Phys. 2007, 12\%, 164321.

(8) Cammi, R.; Corni, S.; Mennucci, B.; Tomasi, J. Electronic Excitation Energies of Molecules in Solution: State Specific and Linear Response Methods for Nonequilibrium Continuum Solvation Models. J. Chem. Phys. 2005, 122, 104513. 
(9) Cammi, R.; Mennucci, B.; Tomasi, J. An Attempt To Bridge the Gap between Computation and Experiment for Nonlinear Optical Properties: Macroscopic Susceptibilities in Solution. J. Phys. Chem. A 2000, 104, 4690-4698.

(10) Mennucci, B.; Cammi, R.; Tomasi, J. Medium Effects on the Properties of Chemical Systems: Electric and Magnetic Response of Donor-Acceptor Systems Within the Polarizable Continuum Model. Int. J. Quantum Chem. 1999, 75, 767-781.

(11) Cammi, R.; Frediani, L.; Mennucci, B.; Tomasi, J.; Ruud, K.; Mikkelsen, K. A SecondOrder, Quadratically Convergent Multiconfigurational Self-Consistent Field Polarizable Continuum Model for Equilibrium and Nonequilibrium Solvation. J. Chem. Phys. 2002, $117,13$.

(12) Cammi, R.; Tomasi, J. Nonequilibrium Solvation Theory for the Polarizable Continuum Model: A New Formulation at the SCF Level with Application to the Case of the Frequency-Dependent Linear Electric Response Function. Int. J. Quantum Chem. $\mathbf{1 9 9 5}, 56,465-474$.

(13) Kim, H. J.; Hynes, J. T. Equilibrium and Nonequilibrium Solvation and Solute Electronic Structure. I. Formulation. J. Chem. Phys. 1990, 93, 5194-5210.

(14) Langlet, J.; Claverie, P.; Caillet, J.; Pullman, A. Improvements of the Continuum model. 1. Application to the Calculation of the Vaporization Thermodynamic Quantities of Nonassociated Liquids. J. Phys. Chem. 1988, 92, 1617-1631.

(15) Amovilli, C.; Mennucci, B. Self-Consistent-Field Calculation of Pauli Repulsion and Dispersion Contributions to the Solvation Free Energy in the Polarizable Continuum Model. J. Phys. Chem. B 1997, 101, 1051-1057.

(16) Cramer, C. J.; Truhlar, D. G. A Universal Approach to Solvation Modeling. Acc. Chem. Res. 2008, 41, 760-768. 
(17) Cramer, C.; Truhlar, D. Implicit Solvation Models: Equilibria, Structure, Spectra, and Dynamics. Chem. Rev. 1999, 99, 2161-2200.

(18) Gratzel, M. Solar Energy Conversion by Dye-Sensitized Photovoltaic Cells. Inorg. Chem. 2005, 44, 6841-6851.

(19) Stillwell, W. An Introduction to Biological Membranes: From Bilayers to Rafts; Academic Press, 2013.

(20) Liss, P. S. The Sea Surface and Global Change; Cambridge University Press, 2008.

(21) Grossfield, A.; Feller, S. E.; Pitman, M. C. Convergence of Molecular Dynamics Simulations of Membrane Proteins. Proteins 2007, 67, 31-40.

(22) Feller, S. E. Molecular Dynamics Simulations of Lipid Bilayers. Curr. Opin. in Coll. E Interf. Science $\mathbf{2 0 0 0 ,}$

(23) Lindahl, E.; Sansom, M. S. P. Membrane Proteins: Molecular Dynamics Simulations. Curr. Opin. Struct. Biol. 2008, 18, 425-431.

(24) Marrink, S. J.; Tieleman, D. P. Perspective on the Martini Model. Chem. Soc. Rev. 2013, 42, 6801.

(25) Shinoda, W.; DeVane, R.; Klein, M. L. Computer Simulation Studies of Self-Assembling Macromolecules. Curr. Opin. Struct. Biol. 2012, 22, 175-186.

(26) Bennun, S. V.; Hoopes, M. I.; Xing, C.; Faller, R. Coarse-Grained Modeling of Lipids. Chem. Phys. Lipids 2009, 159, 59-66.

(27) Ni, Y.; Skinner, J. L. IR and SFG Vibrational Spectroscopy of the Water Bond in the Bulk Liquid and at the Liquid-Vapor Interface, Respectively. J. Chem. Phys. 2015, $143,014502$. 
(28) Warshel, A.; Levitt, M. Theoretical Studies of Enzymic Reactions: Dielectric, Electrostatic and Steric Stabilization of the Carbonium Ion in the Reaction of Lysozyme. J. Molec. Biol. 1976, 103, 227-249.

(29) Gao, J. L. Hybrid Quantum and Molecular Mechanical Simulations: An Alternative Avenue to Solvent Effects in Organic Chemistry. Acc. Chem. Res. 1996, 29, 298-305.

(30) Kongsted, J.; Osted, A.; Mikkelsen, K. V.; Christiansen, O. The QM/MM Approach for Wavefunctions, Energies and Response Functions within Self-Consistent Field and Coupled Cluster Theories. Mol. Phys. 2002, 100, 1813-1828.

(31) Tomasi, J.; Mennucci, B.; Cammi, R. Quantum Mechanical Continuum Solvation Models. Chem. Rev. 2005, 105, 2999-3093.

(32) Frediani, L.; Pomelli, C.; Tomasi, J. n-Alkyl Alcohols at the Water/Vapour and Water/Benzene Interfaces: a Study on Phase Transfer Energies. Phys. Chem. Chem. Phys. 2000, 2, 4876-4883.

(33) Frediani, L.; Cammi, R.; Corni, S.; Tomasi, J. A Polarizable Continuum Model for Molecules at Diffuse Interfaces. J. Chem. Phys. 2004, 120, 3893-3907.

(34) Bondesson, L.; Frediani, L.; Agren, H.; Mennucci, B. Solvation of N-3(-) at the Water Surface: The Polarizable Continuum Model Approach. J. Phys. Chem. B 2006, 110, $11361-11368$.

(35) Mozgawa, K.; Mennucci, B.; Frediani, L. Solvation at Surfaces and Interfaces: A Quantum-Mechanical/Continuum Approach Including Nonelectrostatic Contributions. J. Phys. Chem. C 2014, 118, 4715-4725.

(36) Martins-Costa, M. T. C.; Garcıa-Prieto, F. F.; Ruiz-Lopez, M. F. Reactivity of Alde- 
hydes at the Air-Water Interface. Insights from Molecular Dynamics Simulations and Ab Initio Calculations. Org. Biomol. Chem. 2015, 13, 1673-1679.

(37) Martins-Costa, M. T. C.; Anglada, J. M.; Francisco, J. S.; Ruiz-Lopez, M. F. Reactivity of Volatile Organic Compounds at the Surface of a Water Droplet. J. Am. Chem. Soc. 2012, 134, 11821-11827.

(38) Martins-Costa, M. T. C.; Ruiz-Lopez, M. F. Amino Acid Capture by Aqueous Interfaces. Implications for Biological Uptake. J. Phys. Chem. B 2013, 117, 12469-12474.

(39) Martins-Costa, M. T. C.; Ruiz-Lopez, M. F. Simulation of Amino Acid Diffusion Across Water/Hydrophobic Interfaces. Phys. Chem. Chem. Phys. 2011, 13, 11579-11582.

(40) Andreussi, O.; Dabo, I.; Marzari, N. Revised Self-Consistent Continuum Solvation in Electronic-Structure Calculations. J. Chem. Phys. 2012, 136, 064102.

(41) Cances, E.; Mennucci, B.; Tomasi, J. A New Integral Equation Formalism for the Polarizable Continuum Model: Theoretical Background and Applications to Isotropic and Anisotropic Dielectrics. J. Chem. Phys. 1997, 10\%, 3032-3041.

(42) Chang, T. M.; Dang, L. X. Molecular Dynamics Simulations of CCl4-H2O LiquidLiquid Interface with Polarizable Potential Models. J. Chem. Phys. 1996, 6772-6783.

(43) Dang, L. X.; Chang, T. M. Molecular Dynamics Study of Water Clusters, Liquid, and Liquid-Vapor Interface of Water with Many-Body Potentials. J. Chem. Phys. 1997, $106,8149-8159$.

(44) Zhang, Y.; Feller, S. E.; Brooks, B. R.; Pastor, R. W. Computer Simulation of Liquid/Liquid Interfaces. I. Theory and Application to Octane/Water. J. Chem. Phys. 1995, 103, 10252-10266. 
(45) Mennucci, B.; Cances, E.; Tomasi, J. Evaluation of Solvent Effects in Isotropic and Anisotropic Dielectrics and in Ionic Solutions with a Unified Integral Equation Method: Theoretical Bases, Computational Implementation, and Numerical Applications. J. Phys. Chem. B 1997, 101, 10506-10517.

(46) Di Remigio, R.; Mozgawa, K.; Cao, H.; Weijo, V.; Frediani, L. A Polarizable Continuum Model for Molecules at Spherical Diffuse Interfaces. J. Chem. Phys. 2016, 144, 124103.

(47) Amovilli, C.; McWeeny, R. Perturbation Calculations of Molecular Interaction Energies - an Example, $\mathrm{HF}=\mathrm{HF}$. Chem. Phys. Lett. 1986, 128, 11-17.

(48) Amovilli, C.; McWeeny, R. A Matrix Partitioning Approach to the Calculation of Intermolecular Potentials. General Theory and Some Examples. Chem. Phys. 1990, 140, 343-361.

(49) Amovilli, C. Calculation of the Dispersion Energy Contribution to the Solvation FreeEnergy. Chem. Phys. Lett. 1994, 229, 244-249.

(50) Weijo, V.; Mennucci, B.; Frediani, L. Toward a General Formulation of Dispersion Effects for Solvation Continuum Models. J. Chem. Theory Comput. 2010, 6, 33583364 .

(51) Pierotti, R. Scaled Particle Theory of Aqueous and Non-Aqueous Solutions. Chem. Rev. 1976, 76, 717-726.

(52) Tomasi, J.; Cammi, R.; Mennucci, B. Medium Effects on the Properties of Chemical Systems: An Overview of Recent Formulations in the Polarizable Continuum Model (PCM). Int. J. Quantum Chem. 1999, 75, 783-803.

(53) Cammi, R.; Frediani, L.; Mennucci, B.; Ruud, K. Multiconfigurational Self-Consistent Field Linear Response for the Polarizable Continuum Model: Theory and Application 
to Ground and Excited-State Polarizabilities of para-Nitroaniline in Solution. J. Chem. Phys. 2003, 119, 5818-5827.

(54) Cupellini, L.; Amovilli, C.; Mennucci, B. Electronic Excitations in Nonpolar Solvents: Can the Polarizable Continuum Model Accurately Reproduce Solvent Effects? J. Phys. Chem. B 2015, 119, 8984-8991.

(55) Stephens, P. J.; Devlin, F. J.; Chabalowski, C. F.; Frisch, M. J. Ab-Initio Calculation of Vibrational Absorption and Circular-Dichroism Spectra Using Density-Functional Force-Fields. J. Phys. Chem. 1994, 98, 11623-11627.

(56) BECKE, A. D. Density-Functional Thermochemistry .3. the Role of Exact Exchange. J. Chem. Phys. 1993, 98, 5648-5652.

(57) Frisch, M. J.; Trucks, G. W.; Schlegel, H. B.; Scuseria, G. E.; Robb, M. A.; Cheeseman, J. R.; Scalmani, G.; Barone, V.; Mennucci, B.; Petersson, G. A. et al. Gaussian 09 Revision E.01. Gaussian Inc. Wallingford CT 2009.

(58) Dunning, T. H. Gaussian-Basis Sets for Use in Correlated Molecular Calculations .1. the Atoms Boron Through Neon and Hydrogen. J. Chem. Phys. 1989, 90, 1007-1023.

(59) Parisio, G.; Ferrarini, A. Solute Partitioning into Lipid Bilayers: An Implicit Model for Nonuniform and Ordered Environment. J. Chem. Theory and Comput. 2010, 6, $2267-2280$.

(60) Leopardi, P. A Partition of the Unit Sphere into Regions of Equal Area and Small Diameter. ETNA 2006, 25, 309-327.

(61) Martins-Costa, M. T. C.; Ruiz-Lopez, M. F. Solvation Effects on Electronic Polarization and Reactivity Indices at the Air-Water Interface: Insights from a Theoretical Study of Cyanophenols. Theor. Chem. Acc. 2015, 137, 17. 
(62) Canuto, S.; Coutinho, K. From Hydrogen Bond to Bulk: Solvation Analysis of the $n \rightarrow \pi^{*}$ Transition of Formaldehyde in Water. Int. J. Quantum Chem. 2000, 77, 192198. 


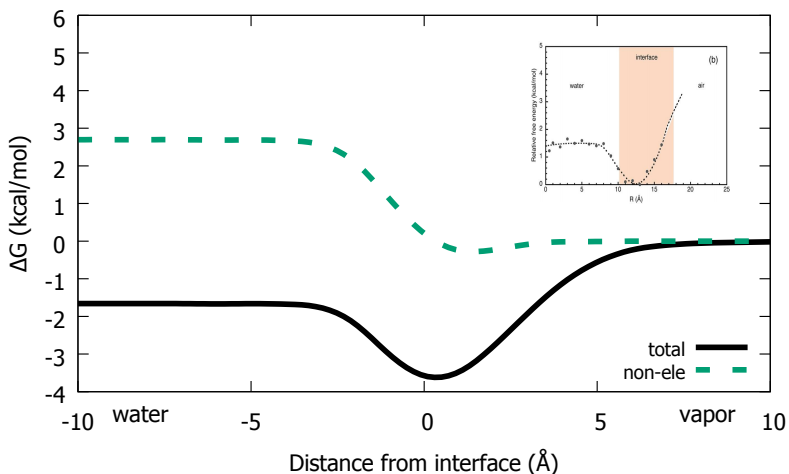

(a) Total solvation energy (full) and non-electrostatic terms (dashed)

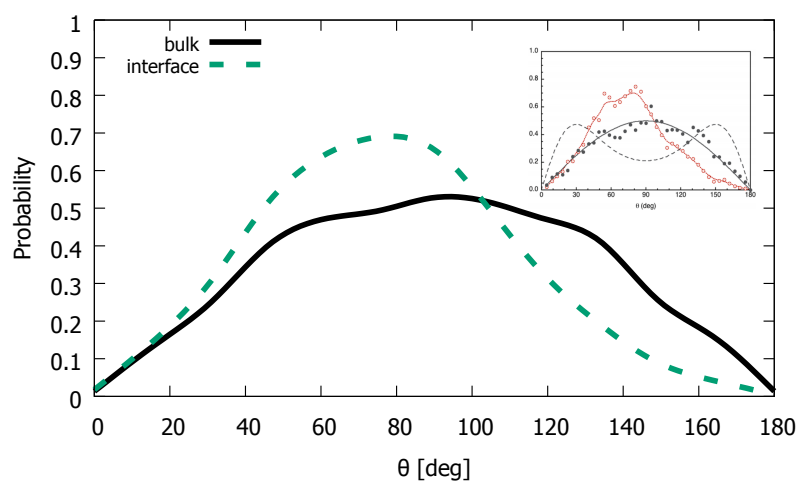

(c) angular distribution of formaldehyde in bulk (full) and at the water surface (dashed)

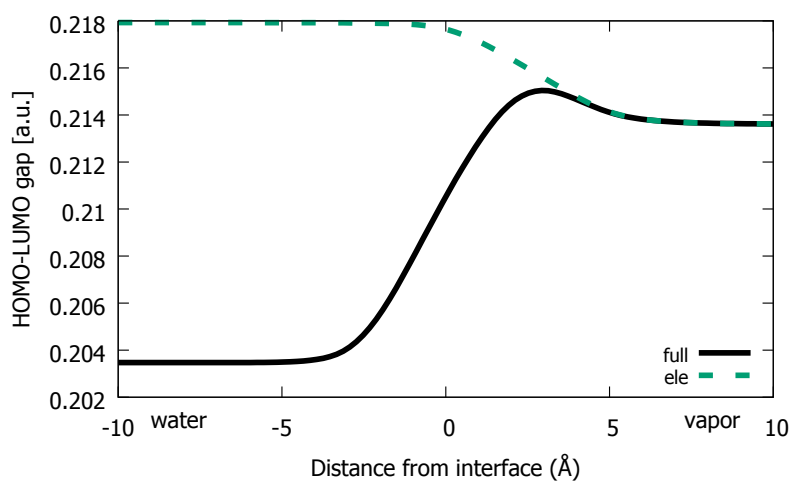

(e) HOMO-LUMO gap of formaldehyde across the water surface

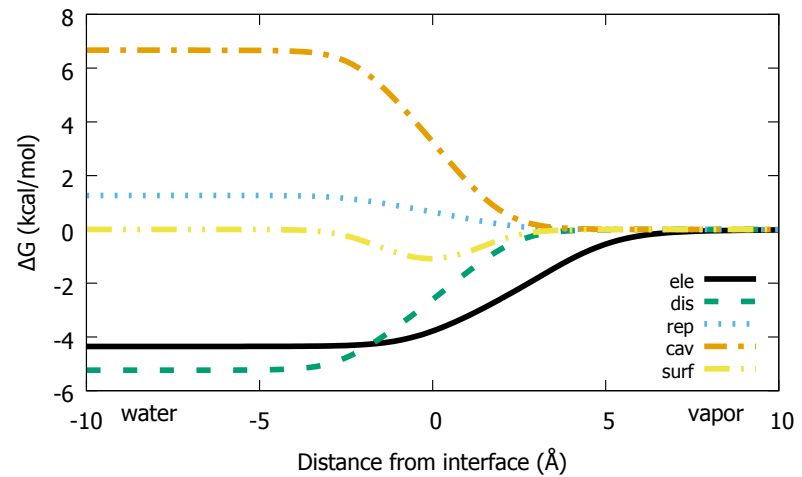

(b) Separated contributions to $\Delta G_{\mathrm{sol}}$ : electrostatics (full), dispersion (dashed), repulsion (dotted), cavitation (dashdot), surface (dash-2 dots).

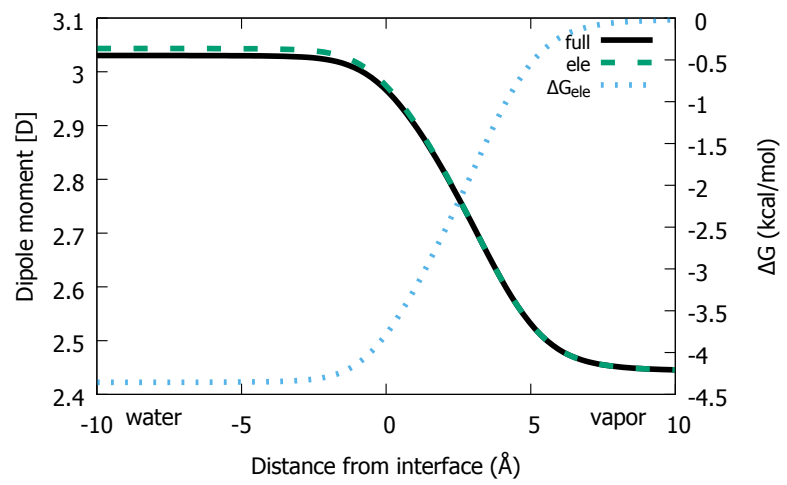

(d) Dipole moment of formaldehyde across the water surface

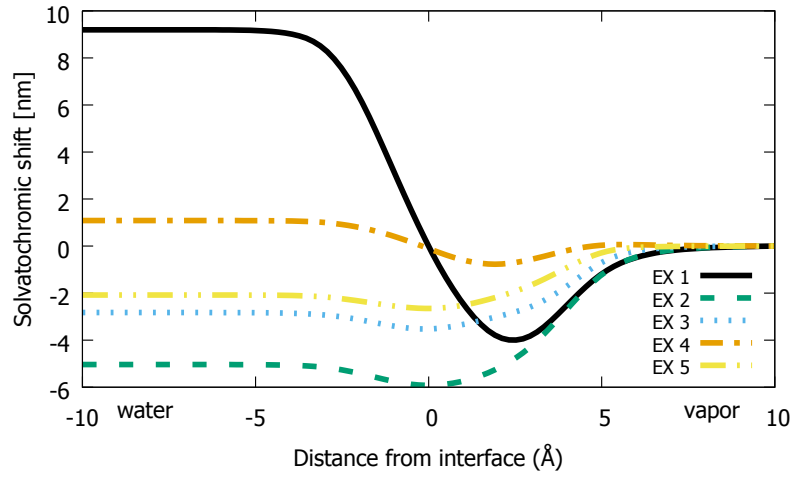

(f) Solvatochromic shifts of the first five excitation energies of formaldehyde across the water surface

Figure 1: Solvation process of formaldehyde at the water surface. The preferential solvation at the water surface is due to the interplay of the different solvation energy contributions (see panels (a) and (b)) and the angular distribution at the water surface shows a preferential orientation with the carbonyl pointing towards the water surface as observed in Ref. ${ }^{37}$ (inset reprinted with permission from Ref., ${ }^{37}$ copyright 2012 American Chemical Society.). The dipole moment (c) is only sensitive to the electrostatic environment whereas the HOMO-LUMO gap (e) and the solvatochromic shifts (f) are significantly affected by dispersion and repulsion. 


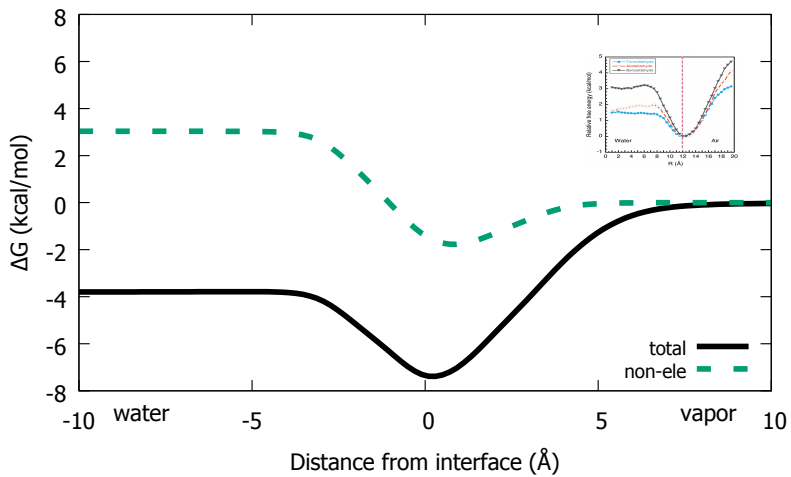

(a) Total solvation energy (full) and non-electrostatic terms (dashed)

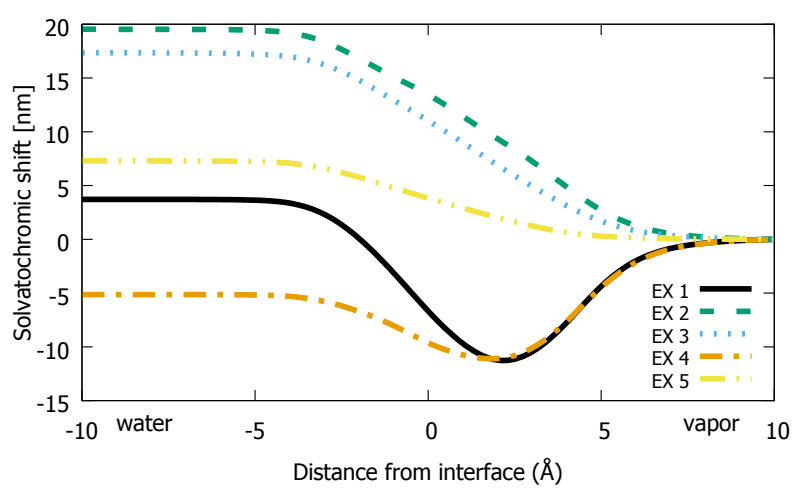

(c) Solvatochromic shifts of the first five excitation energies of benzaldehyde across the water surface

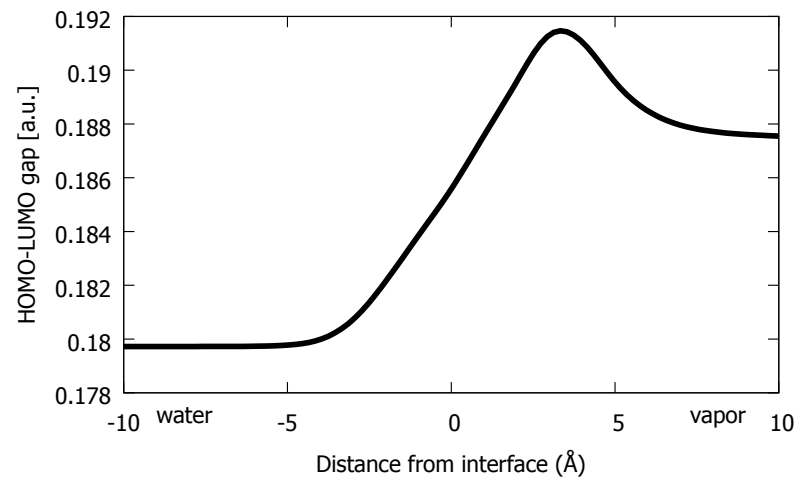

(b) HOMO-LUMO gap of benzaldehyde across the water surface

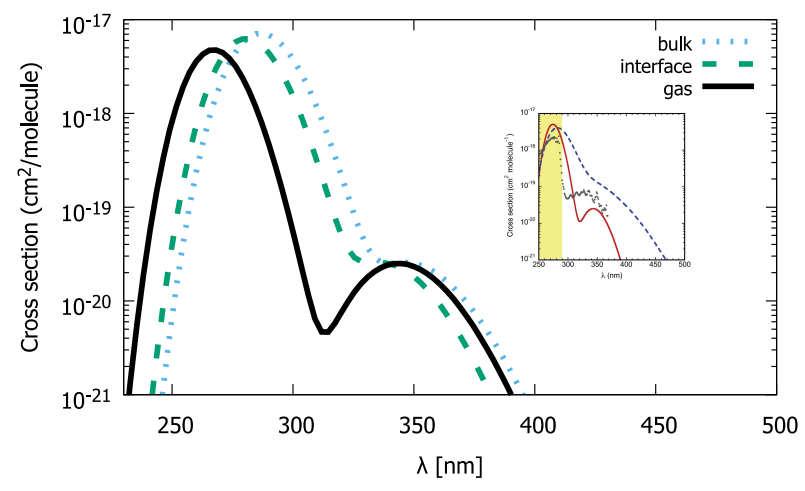

(d) Simulated absorption spectrum of formaldehyde in gas (full), at the water surface (dashed) and in bulk (dotted)

Figure 2: Solvation process of benzaldehyde at the water-vapor water surface. The preferential solvation at the water surface (a) is accompained by strong non-electrostatic effects both on the HOMO-LUMO gap and on the excitation energies. The simulated UV-Vis spectrum shows how the absorption is affected by the the different environments: gas-phase (solid), water surface (dashed), water bulk (dotted line) (insets in (a) and (d) are reproduced with permission from Ref. ${ }^{36}$ Copyright: The Royal Society of Chemistry). 


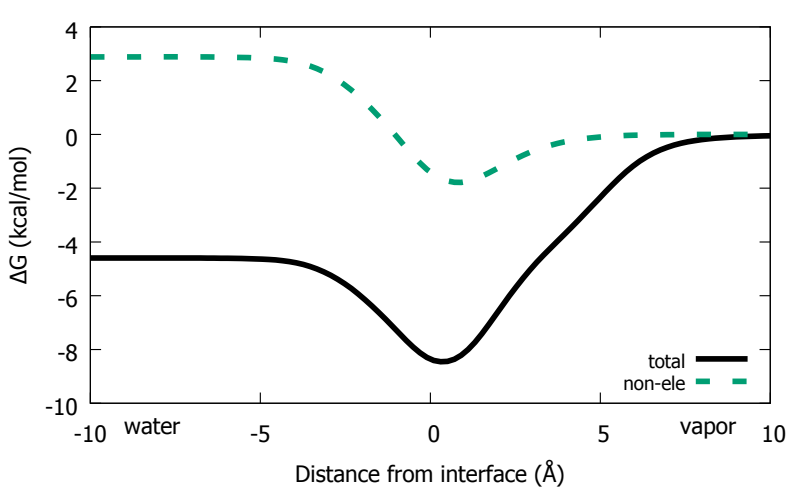

(a) Total solvation energy (full) and non-electrostatic terms (dashed) of o-cyanophenol across the water surface

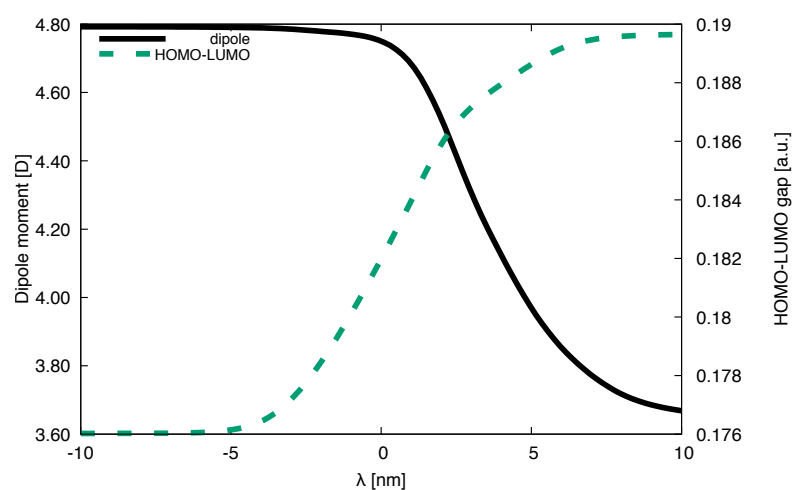

(b) Dipole moment and HOMO-LUMO gap of o-cyanophenol across the water surface

Figure 3: Solvation process of o-cyanophenol at the water surface. The preferential solvation at the water surface is due to the interplay of the different solvation energy contributions (a). The variations in the dipole moment is again an electrostatic effect, whereas the the HOMO-LUMO variations are caused by non-electrostatic effects (b). 


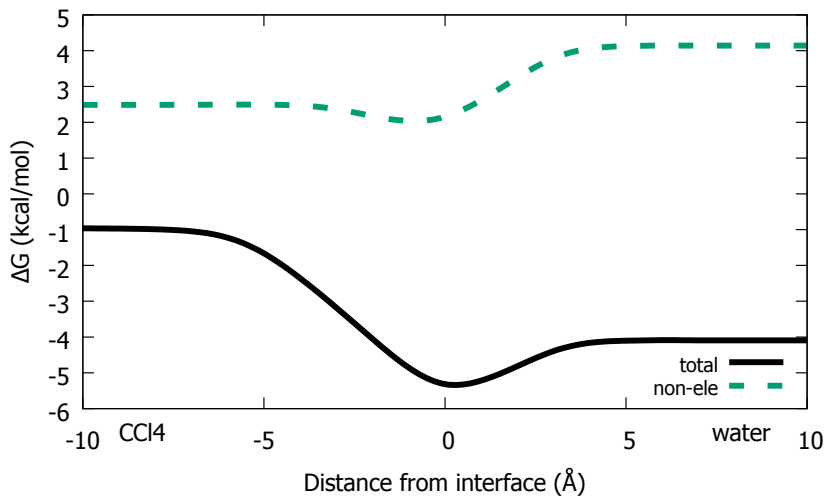

(a) Glycine: solvation energy profile of the N1 form across the $\mathrm{H}_{2} \mathrm{O}-\mathrm{CCl}_{4}$ interface

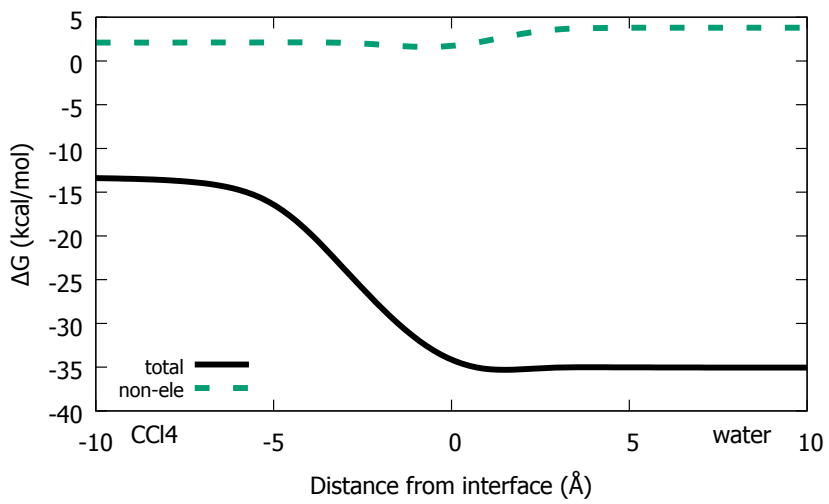

(c) Glycine: solvation energy profile of the ZW form across the $\mathrm{H}_{2} \mathrm{O}-\mathrm{CCl}_{4}$ interface

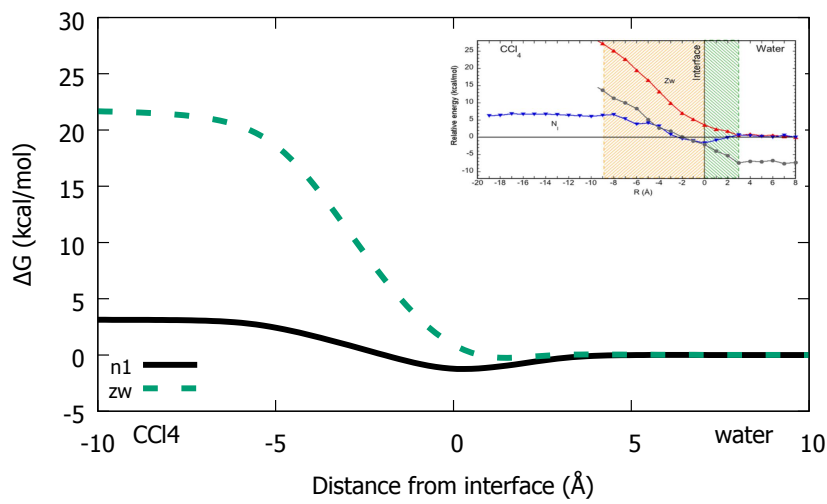

(e) Glycine: comparison with Ref. ${ }^{38}$

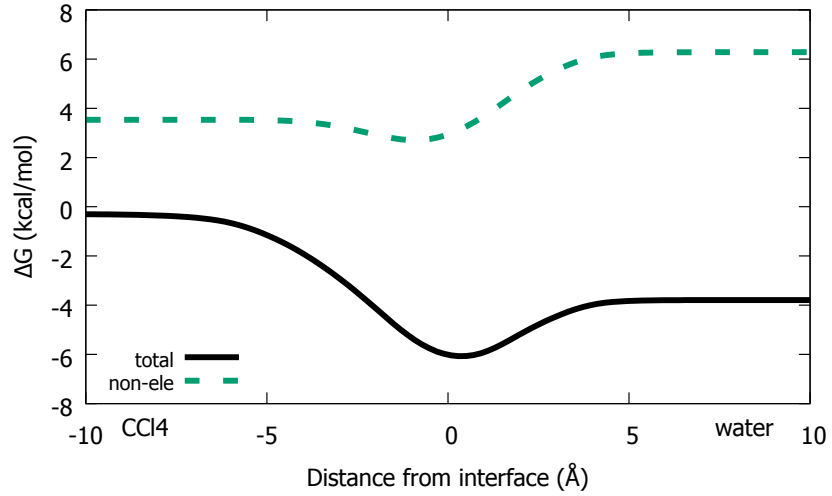

(b) Valine: solvation energy profile of the $\mathrm{N} 1$ form across the $\mathrm{H}_{2} \mathrm{O}-\mathrm{CCl}_{4}$ interface

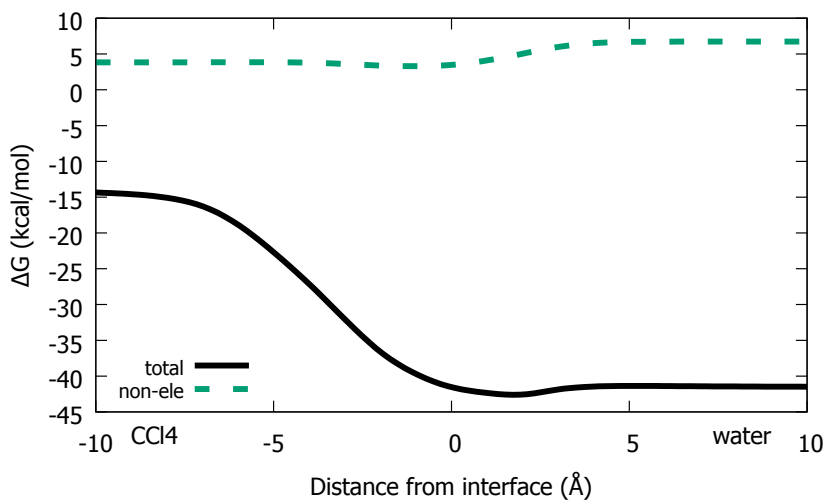

(d) Valine: solvation energy profile of the ZW form across the $\mathrm{H}_{2} \mathrm{O}-\mathrm{CCl}_{4}$ interface

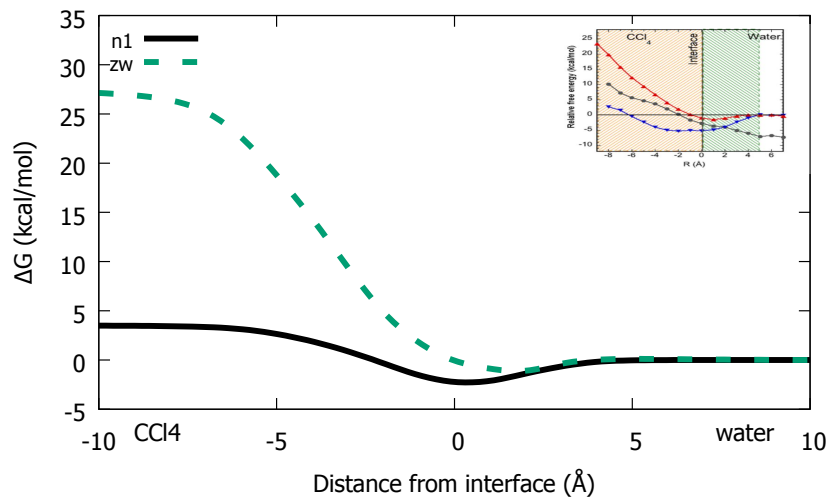

(f) Valine: comparison with Ref. ${ }^{38}$

Figure 4: Free energy of solvation profiles for glycine (left side and valine (right side), both in N1 and ZW forms. The top-row displays the results for the neutral form, the middle row for the zwitterionic ones, and the bottom panels report a comparison with Ref. ${ }^{38}$ (inset reproduced with permission, copyright American Chemical Society). For the sake of comparison the results in the bottom panel are reported taking species solvated in water as the reference point. All values $\mathrm{i}$ $\mathrm{kcal} / \mathrm{mol}$. 


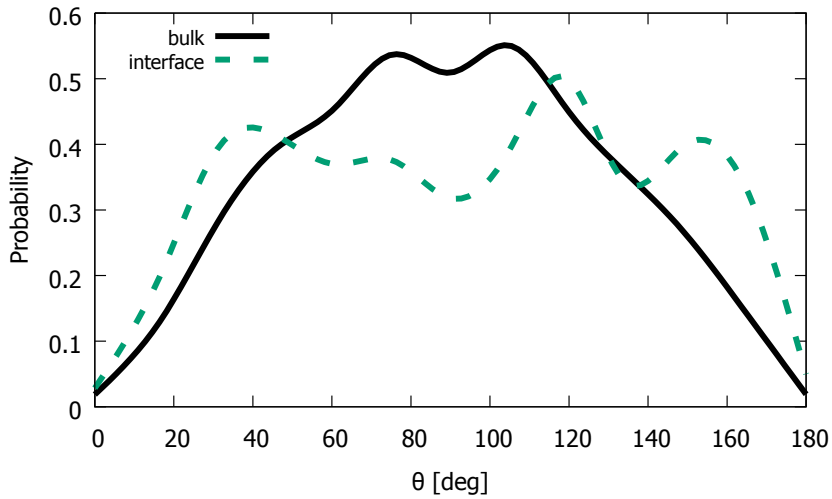

(a) glycine $\mathrm{N} 1$

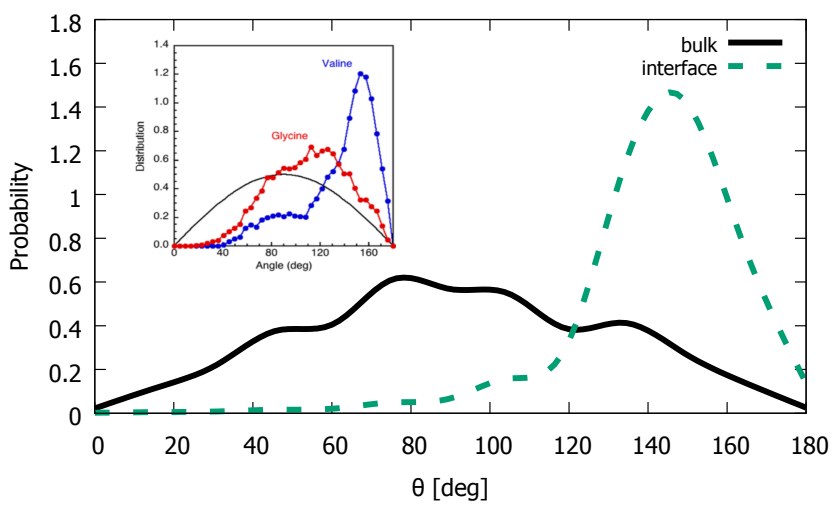

(c) valine N1

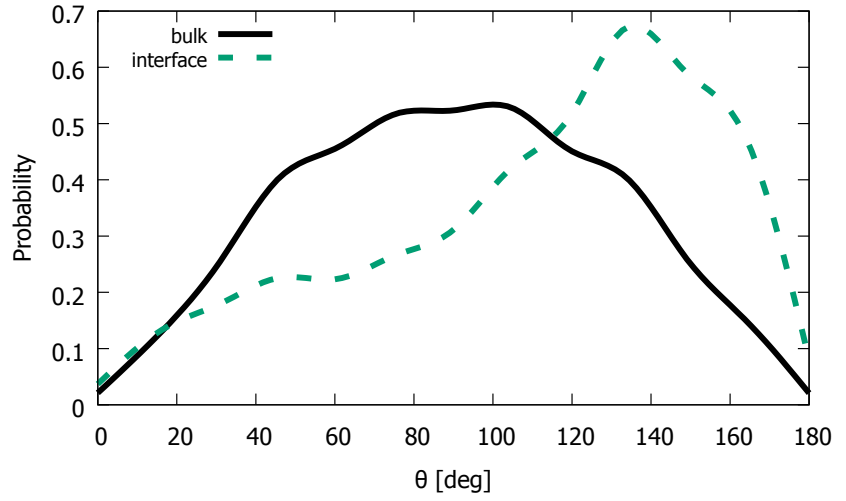

(b) glycine ZW

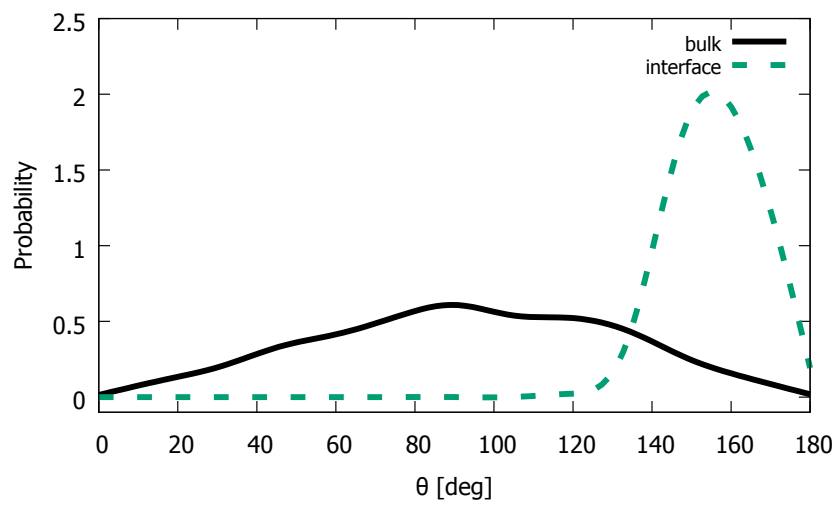

(d) valine $\mathrm{ZW}$

Figure 5: Angular distribution for glycine (top) and valine (bottom), both in N1 (left) and ZW (right) forms, at the $\mathrm{CCl}_{4}$-water interface and in bulk. The inset from $\mathrm{Ref}^{38}$ presents the angular distributions for N1 forms (reprinted with permission, copyright 2013 American Chemical Society.) 
Graphical TOC Entry

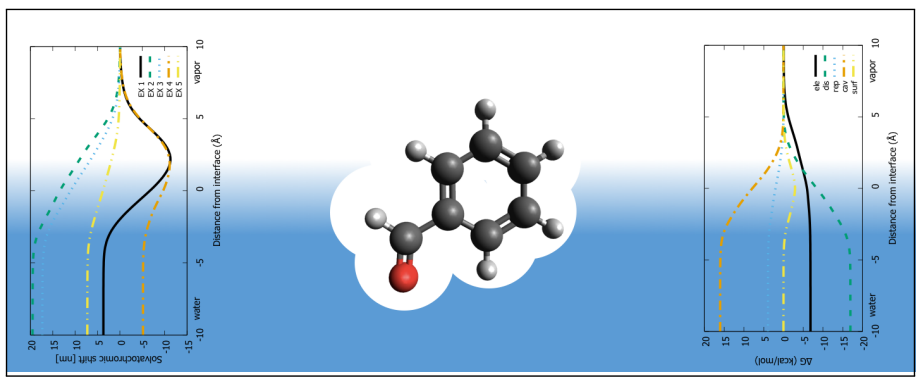

